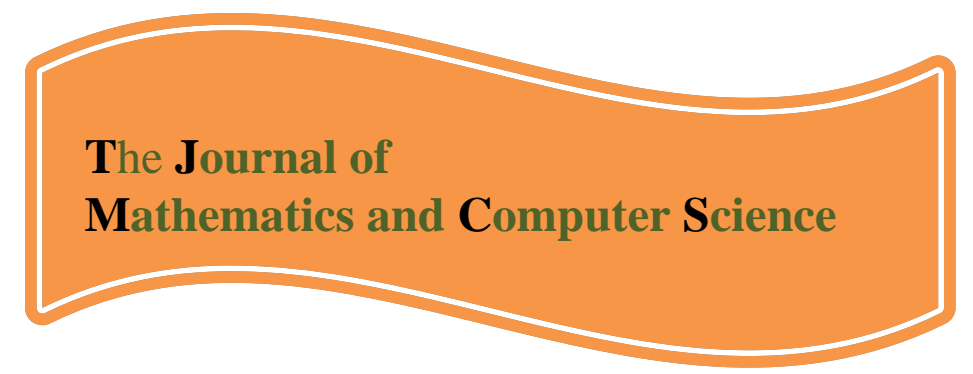

Available online at

http://www.TJMCS.com

The Journal of Mathematics and Computer Science Vol .1 No.4 (2010) 333-338

\title{
Fuzzy Material Requirements Planning
}

\author{
Hamid Reza Feili ${ }^{1 *}$, Mahzad Shakeri Moghaddam² ${ }^{2}$ Rezvan Zahmatkesh $^{3}$ \\ hrfeili@gmail.com \\ mahzad.shakeri@gmail.com \\ rezvanz@aol.com
}

Received: August 2010, Revised: October 2010

Online Publication: December 2010

\begin{abstract}
:
Various modeling techniques based on possibility distribution function has been applied successfully in a wide range of issues related to production planning. However, the possibility distribution function derived from the records recorded in the organization are not always available or changes in production environment may cause a variety of changes and volatilities in model and make it unreliable; changes such as: market demand, changes in types of production costs, capacity, resources, and administrative constraints.

In this study fuzzy sets theory has been combined with material requirements planning (MRP). It is noteworthy that material requirements planning (MRP) is one of the most commonly used subdivision of production planning that combining it with fuzzy theory can be applied to develop decision making systems and make them more efficient. In addition, the uncertainties of industrial environments can be exerted properly into the production decision-making.
\end{abstract}

Keywords: Production Planning, Material Requirements Planning, Uncertainty, Fuzzy

\section{Introduction}

There are many forms of uncertainty in industrial decision environments, which can affect our decision making during production planning processes.

1*. Assistant Professor, Industrial Engineering, Alzahra University

2. B.S, Industrial Engineering, Alzahra University

3. B.S, Industrial Engineering, Alzahra University 
One of the key sources of uncertainty in any production-distribution system is the product demand $[13,11,6]$. The market demand is composed of firm orders which are known at the beginning of each planning horizon and demand forecasts which are based on records of previous sales. The capacity data neither is a crisp value because during the production processes unforeseen events such as breakdowns, faulty production or preparation delays can happen. Also, planning costs could not be measured easily

since they imply a great use of the human perception for their valuation. For instance, in some manufacturing environments the planners use an average production cost per hour perceived in the calculations of the unitary production costs, what makes these costs fuzzy. The costs of under time and overtime of the productive resources neither could be exact because the manpower can change or because mishaps or production shortcomings can take place. On the other hand, the demand backlog cost is fuzzy in its composition. This cost consists so much of the administrative cost of managing the backlog of orders as of the cost due to the loss of the clients. This type of cost is commonly estimated by using human experiences.

Along the years there have been many researches and applications aimed to model the uncertainty in production planning problems [9]. Uncertainty can be present as randomness, fuzziness and/or lack of knowledge or epistemic uncertainty (see [2]).

Here, we model a production planning problem under uncertainty with fuzzy constraints, taking into account the fuzziness in the demand, the available capacity and the aspiration level of costs and fuzzy coefficients, the lack of knowledge of the demand backlog costs and the required capacity, based on fuzzy linear programming.

\section{Problem Description}

Different modeling techniques based on possibility distribution function has been applied successfully in a wide range of issues related to production planning $[10,4,5,6,8,12]$. However, the possibility distribution function derived from the records recorded in the organization are not always available or changes in production environment may cause a variety of changes and volatilities in model and make it unreliable; changes such as: market demand, changes in types of production costs, capacity, resources, and administrative constraints.

In these situations, the fuzzy set theory has been used to model systems that are difficult to define accurately $[1,3,14]$. This theory represents an attractive tool to support the production

planning research when the dynamics of the manufacturing environment limits the specification of the model objectives, constraints and parameters.

To distinguish between fuzziness or flexibility in constraints and goals and lack of knowledge of the data or epistemic uncertainty, it is necessary to know that flexibility is modeled by fuzzy constraints while epistemic uncertainty is modeled by fuzzy coefficients through possibilistic programming.

Material requirements planning (MRP) can be modeled and analyzed as fuzzy nature and structure. Issues such as: the level of customer satisfaction, level of changes in orders, lead times, costs of changes, level of other customers' satisfaction whose delivery date may get delayed cause of changes in orders, rate of competitors; service, market changes and etc. can be defined as fuzzy parameters and the model can be analyzed as fuzzy analysis[16]. 
The model proposed in this section considers the possible lack of knowledge in data and existent fuzziness jointly in the lack of knowledge of the demand backlog costs and the required capacity. Decision variables and parameters for the fuzzy model and the fuzzy mathematical programming model are defined in Tables 1-5.

\begin{tabular}{|c|l|}
\multicolumn{1}{c|}{ symbol } & meaning \\
\hline$T$ & Number of periods in the planning horizon $(t=1, \ldots, T)$ \\
\hline$I$ & Number of products $(i=1, \ldots, I)$ \\
\hline$J$ & Number of the parent products in the bill of materials $(j=1, \ldots, J)$ \\
\hline$R$ & Number of resources $(r=1, \ldots, R)$ \\
\hline
\end{tabular}

Table 2.Decision variables

\begin{tabular}{|c|l|}
\multicolumn{1}{|c|}{ symbol } & meaning \\
\hline$Q_{i t}$ & Quantity to produce of the product $i$ on period $t$ \\
\hline$S_{i t}$ & Inventory of the product $i$ at the end of period $t$ \\
\hline$B_{i t}$ & Demand backlog of the product $i$ at the end of period $t$ \\
\hline$U_{r t}$ & Undertime hours of the resource $r$ on period $t$ \\
\hline$O_{r t}$ & Overtime hours of the resource r on period $t$ \\
\hline
\end{tabular}

Table 3.Objective function cost coefficients

\begin{tabular}{|c|l|}
\multicolumn{1}{|c|}{ symbol } & meaning \\
\hline$c p_{i}$ & Variable cost of production per unit of the product $i$ \\
\hline$c i_{i}$ & Inventory cost per unit of the product $i$ \\
\hline$<c r \tilde{d}_{i}, d 1_{i}>$ & Fuzzy cost due to the demand backlog per unit of the product $i$ \\
\hline$c t o c_{r t}$ & Undertime hour cost of the resource $r$ on period $t$ \\
\hline$c t e x_{r t}$ & Overtime hour cost of the resource $r$ on period $t$ \\
\hline
\end{tabular}

Table 4.Technological coefficients

\begin{tabular}{|c|l|}
\multicolumn{1}{c|}{ symbol } & meaning \\
\hline$<A \widetilde{R}_{i r}, d 4_{i r}>$ & Fuzzy required time of the resource $r$ per unit of production of the product $i$ \\
\hline$\left[C A \tilde{P}_{r t}, C A \tilde{P}_{r t}+p 3_{r t}\right]$ & Fuzzy available capacity of the resource $r$ in the period $t$ \\
\hline
\end{tabular}

Table 5.Data

\begin{tabular}{|c|l|}
\multicolumn{1}{c|}{ symbol } & meaning \\
\hline$\left[d_{i t}, d_{i t}+p 2_{i t}\right]$ & Fuzzy demand of the product $i$ on period $t$ \\
\hline$\alpha_{i j}$ & Required quantity of $i$ to produce an unit of the product $j$ \\
\hline$T S_{i}$ & Lead time of the product $i$ \\
\hline
\end{tabular}




\begin{tabular}{|c|l|}
\hline$S_{i 0}$ & Inventory of the product $i$ on period 0 \\
\hline$R d_{i 0}$ & Demand backlog of the product $i$ on period 0 \\
\hline$R P_{i t}$ & Programmed receptions of the product $i$ on period $t$ \\
\hline$[z 1, z 1+p 1]$ & Fuzzy desired level of total costs \\
\hline
\end{tabular}

We have used the symbol <,>to represent a symmetric triangular fuzzy number following the notation proposed by Inuiguchi et al. [15]. It is not really an interval, represented by the symbol [, ], with a minimum and a maximum value of the tolerance interval. The left value inside of the symbol <,>indicates "the most possible value" of the fuzzy set and the right value defines the maximum deviation from "the most possible value" to "the less possible values". For instance, a symmetric triangular fuzzy number given by $<60,5>$ corresponds to the linguistic expression "about 60" and represents that the most possible value is 60 and the less possible values are 55 and 65.

The fuzzy model for MRP with uncertainty in data and fuzzy aspirations is formulated as follows:

$$
\begin{aligned}
& \sum_{i=1}^{I} \sum_{t=1}^{T}\left(c p_{i} Q_{i t}+c i_{i} S_{i t}+c r \tilde{d}_{i} d_{i t}\right)+\sum_{r=1}^{R} \sum_{t=1}^{T}\left(\operatorname{cotoc}_{r t} U_{r t}+\operatorname{ctex}_{r t} O_{r t}\right)<\approx z_{1} \\
& S_{i, t-1}+Q_{i, t}-T s_{i}+R Q_{i t}-S_{i, t}-B_{i, t-1}-\sum_{j=1}^{I} \propto_{i j}\left(Q_{j t}+R Q_{j t}\right)+B_{i t}<\approx d_{i t} \quad, i=1, \ldots . I, t \\
& =1, \ldots . T \\
& S_{i, t-1}+Q_{t, t-T s i}-T s_{i}+P R_{i t}-S_{i t}-B_{i, t-1}-\sum_{j=1}^{I} \propto_{i j}\left(Q_{j t}+R Q_{j t}\right)+B_{i t}>\approx d_{i t}, i=1, \ldots I t \\
& =1, \ldots T \\
& \sum_{i=1}^{I} A \tilde{R}_{i r} Q_{i t}+U_{r t}-O_{r t}<\approx C A \tilde{P}_{r t}, \quad r=1, \ldots . R, t=1, \ldots . T \\
& \sum_{i=1}^{I} A \tilde{R}_{i r} Q_{i t}+U_{r t}-O_{r t}>\approx C A \tilde{P}_{r t}, \quad r=1, \ldots . R, t=1, \ldots . T \\
& B_{i t}=0 \quad i=1, \ldots . I \\
& Q_{i t}, S_{i t}, B_{i t}, U_{r t}, O_{r t} \geq 0 \quad i=1, \ldots . I, r=1, \ldots R, t=1, \ldots . T
\end{aligned}
$$

where the cost constraint (1) and capacity constraints (4) and (5) have fuzzy coefficients represented by symmetric triangular fuzzy numbers and cost, demand and capacity constraints (1)-(5) are described explicitly as fuzzy inequalities specified by their corresponding membership function.

Eq. (1) shows the aspiration level, $z 1$, of total cost to be reached. The total cost is formed by the production costs, inventory costs, demand backlog costs, and costs of undertime and overtime of the productive resources. Most of these costs cannot be measured easily since they imply a great use of the human perception for their valuation. Therefore, the aspiration level of total cost is fuzzy because of these possible fuzzy costs. Also, the model includes a plan to satisfy the backlog 
of demand penalized with a cost, cr $\tilde{i}$. It is assumed that this cost is uncertain and it is represented by a symmetric triangular fuzzy number.

The balance equations for the inventory are given by the group of fuzzy constraints (2) and (3). These equations take into account the backlog of demand which behave as a negative inventory. It is important to highlight the consideration of the parameter RQit which guarantees the continuity of the MRP along the successive explosions carried out during a given planning horizon. Also, it is considered fuzziness in the market demand represented by fuzzy constraints. The symbol _ represents the fuzzy version of _ and means "essentially less than or similar to". These constraints show that the planner wants to make the left-hand side of the constraints, the satisfied demand, smaller or similar to the right-hand side, the foreseen demand, "if it is possible". In the same manner, the constraints of the type _ represent the fuzzy version of _ and mean "considerably bigger or similar to". These constraints show that the planner wants to make the left-hand side, the satisfied demand, of the constraints bigger or similar to the right-hand side, the foreseen demand, "if it is possible".

Fuzzy Eqs. (3) and (4) consider the limits of capacity of the productive resources. The decision variables Urt and Ort are not limited by any established parameter but are penalized with the corresponding costs in the objective function. It is considered fuzziness in the available capacity represented by fuzzy constraints. Also, the required capacity, $A \tilde{R} i r$, is considered uncertain data modeled by a symmetric triangular fuzzy number.

A constraint has also been considered (6) to finish with the delays in the last period $T$ of the planning horizon. The model also contemplates the non-negativity constraints (7) for the decision variables. Finally, the decision variables Qit, Sit and Bit will be defined as continuous or integer variables depending on the manufacturing environment where the model is applied.

\section{Conclusion}

Decision making during production planning process under conditions of uncertainty needs a model in which parameters can be defined as uncertain ones. For improving such situation, a fuzzy production planning model is developed in this paper.

The proposed fuzzy model has tried to soften the rigid requirements in the traditional linear programming models making fuzzier the perception based on uncertainties. It is true that, in general, models with fuzzy constraints have got a better behavior than the model with fuzzy constraints and fuzzy coefficients but with the proposed model we can introduce to the model both types of uncertainties, fuzziness and lack of knowledge or epistemic uncertainty.

Since resolving the model by conventional scientific methods in applicable soft wares such as Lingo is time consuming, in next attempt we will research for an appropriate Meta heuristic approach to reduce the computer time -which is very important in executive environments- to solve the model.

\section{References}

[1] R.E. Bellman, L.A. Zadeh, Decision making in a fuzzy environment, Management Sci. 17 (1970) 141-164.

[2] D. Dubois, H. Fargier, P. Fortemps, Fuzzy scheduling: modelling flexible constraints vs. coping with incomplete knowledge, European J. Oper. Res. 147 (2003) 231-252.

[3] D. Dubois, H. Prade, Fuzzy Sets and Systems: Theory and Applications, NewYork, London, Toronto, 1980. 
[4] L.F. Escudero, P.V. Kamesam, A.J. King, R.J.B.Wets, Production planning via scenario modelling, Ann. Oper. Res. 43 (1993) 311-335.

[5] H. Gfrerer, G. Zäpfel, Hierarchical model for production planning in the case of uncertain demand, European J. Oper. Res. 86 (1995) 142-161.

[6] A. Gupta, C.D. Maranas, Managing demand uncertainty in supply chain planning, Comput. Chemical Eng. 27 (2003) 1216-1227.

[7] M. Inuiguchi, M. Sakawa, Y. Kume, The usefulness of possibilistic programming in production planning problems, Internat. J. Production Econom. 33 (1994) 45-52.

[8] D. Kira, M. Kusy, I. Rakita, A stochastic linear programming approach to hierarchical production planning, J. OR Soc. 48 (1997) 207-211.

[9] J. Mula, R. Poler, J.P. García-Sabater, F.C. Lario, Models for production planning under uncertainty: a review, Internat. J. Production Econom. 103 (2006) 271-285.

[10] D. Paraskevopoulos, E. Karakitsos, B. Rustem, Robust capacity planning under uncertainty, Management Sci. 37 (1991) 787-800.

[11] S.B. Perkov, C.D. Maranas, Multiperiod planning and scheduling of multiproduct batch plants under demand uncertainty, Industrial Eng. Chem. Res. 36 (1997) 48-64.

[12] M.S. Sodhi, Managing demand risk in tactical supply chain planning for a global consumer electronics company, Production Oper.Management 14 (2005) 69-79.

[13] D.C. Whybark, J.G.Williams, Material requirements planning under uncertainty, Decision Sci. 7 (1976) 595-606.

[14] H.J. Zimmermann, Fuzzy Set Theory and its Applications, third ed., Kluwer Academic Publishers, Dordrecht, 1996.

[15] K.V. Donselaar, J.V. Nieuwenhof, J. Visschers, The impact of material coordination concepts on planning stability in supply chains, Internat. J. Production Econom. 68 (2000) 169-176.

[16] H.Shavandi,Fuzzy Sets Theory and its Application in Industrial Engineering and Management,Iran,2006 